\title{
Effect of nano-formulated antioxidant on development of renal fibrosis induced by cisplatin
}

\author{
Faten Zahran ${ }^{4}$, Ahmed Nabil $^{5}$, Amro El-Karef ${ }^{3}$, Ibrahim M. El-Sherbiny ${ }^{6}$ and Mohamed Sobh ${ }^{1,2}$ \\ ${ }^{1}$ Urology and Nephrology Center, Faculty of Medicine, Mansoura University, Mansoura, Egypt \\ ${ }^{2}$ Medical Experimental Research Center (MERC), Faculty of Medicine, Mansoura University, Mansoura, Egypt \\ ${ }^{3}$ Pathology Department, Faculty of Medicine, Mansoura University, Mansoura, Egypt \\ ${ }^{4}$ Biochemistry Department, Faculty of Science, Zagazig University, Egypt \\ ${ }^{5}$ Faculty of Postgraduate Studies for Advanced Science Beni-Suef University, Egypt \\ ${ }^{6}$ Zewail City for Science and Technology, Egypt
}

\begin{abstract}
Cisplatin causes renal fibrosis, but the development mechanism of interstitial fibrosis is not yet understood. We examined the effect of nano form antioxidant, $\mathrm{N}$, N'diphenyl-1, 4-phenylenediamine (Nano-DPPD), on development of interstitial fibrosis induced by cisplatin. Cisplatin increased blood urea nitrogen (BUN), serum creatinine, serum magnesium and serum chemoattractant protein (MCP-1) on the other hand it decreased creatinine clearance levels. Also administration of cisplatin made many pathological abnormalities represented in increasing collagen deposition, $\alpha$-smooth muscle actin, Ki67 and renal tubular injury. Administration of Nano-DPPD, which was started at 3 days after cisplatin treatment, significantly inhibited the increase in renal collagen contents and the expansion of the interstitial fibrosis area. These results indicate that anti-fibrotic action of Nano-DPPD is not secondary due to the inhibition of acute renal injury but is rather a direct effect on renal fibrogenesis. Nano-DPPD prevent the infiltration of macrophages by cisplatin, suggesting that anti-fibrotic action of Nano-DPPD was not mediated by the inhibition of inflammatory cellular influx. It is suggested that reactive oxygen species and collagen deposition are involved in cisplatin-induced renal interstitial fibrosis.
\end{abstract}

\section{Introduction}

Cis-Diamminedichloroplatinum (II) (cisplatin) is antitumor agent for testis, bladder, head and neck, lung, and ovarian cancers. Cisplatin causes severe side effects such as renal impairment, gastrointestinal toxicity, and ototoxicity. In particular, its use is limited due to the induced renal fibrosis with tubular necrosis in the kidney [1,2]. The acute renal impairment induced by antitumor cisplatin has been described in animals and humans. Antioxidants that acts as radical scavengers prevented renal impairment induced by cisplatin administration in rats $[3,4]$.also, cisplatin increased the lipid peroxidation and collagen deposition in rat renal tissues [5] and the synthesis of hydrogen peroxide in renal cells [6]. These findings suggest that reactive oxygen species (ROS) and collagen deposition play an important role in the pathogenesis of cisplatin-related renal fibrosis. It is known that cisplatin causes chronic interstitial nephritis with interstitial fibrosis in humans [7]. In experimental animals, cisplatin causes renal interstitial fibrosis in the long term [8]. Interstitial fibrosis is a common lesion in most chronic kidney diseases [9]. The development of interstitial fibrosis is thought to cause irreversible renal dysfunction [9]. However, a few reports have described the relationship between a renal function and development of fibrosis by cisplatin. Collagen deposition played an important role in renal fibrosis by cisplatin, we investigated the role of collagen in the development of fibrosis induced by cisplatin in kidney. We have reported that nano-antioxidant, N, N'-diphenyl-1,4phenylenediamine ( Nano-DPPD), prevented the increases in content of collagen and $\alpha$-SMA and nephrotoxicity induced by cisplatin [10]. So, we determined the effect of Nano-DPPD on the development of fibrosis induced by cisplatin in rats. In this case, interstitial fibrosis may be suppressed merely due to the inhibition of cisplatin-induced renal injury by the antioxidant. To eliminate an effect of antioxidant on renal injury in the progression of fibrosis by cisplatin, we administered an antioxidant to the rats at a time that does not influence renal fibrosis induced by cisplatin. Administration of antioxidant as nano - form was useful as it increased the drug half life time in plasma so single dose of nano-DPPD was enough in renal fibrosis inhibition.

\section{Aim of the work}


diphenyl-1, 4-phenylenediamine on amelioration of renal fibrosis and restoration of renal function in cisplatin induced nephrotoxicity in rat model.

\section{Materials and methods}

\section{Experimental animals}

The study was carried on 45 male Sprague-Dawley rats (body weight

Correspondence to: Dr. Ahmed Nabil, Faculty of Postgraduate Studies for Advanced Science Beni-Suef University, Egypt, Tel: (+20)1000618349, E-mail: drnabil_100@hotmail.com

Key words: cisplatin, renal injury, Nano-DPPD, interstitial fibrosis, oxidative stress

Received: May 14, 2016; Accepted: May 27, 2016; Published: May 31, 2016 
$170-220 \mathrm{~g})$ Rats were bred and maintained in an air-conditioned animal house with specific pathogen free conditions, and were subjected to a 12:12-h daylight/darkness and allowed unlimited access to chow and water. All the ethical protocols for animal treatment were followed and supervised by the animal facilities, Medical Experimental Research Centre, Faculty of Medicine, Mansoura University. Rats were divided into 3 groups as follow:

(1) Control group, where 15 Rats were received intravenous saline instead of cisplatin and served as the control.

(2) Cisplatin group, where 15 Rats were received cisplatin (6 mg/ $\mathrm{kg}$, i.p.) at the start of experiments.

(3) Nano-formulated DPPD group, where 10 Rats were received cisplatin $(6 \mathrm{mg} / \mathrm{kg}$, i.p. $)$ at the start of experiments and Three days after cisplatin administration, rats were given Nano-formulated DPPD (0.5 $\mathrm{g} / \mathrm{kg}$, i.p.) single dose.

At 14 days after cisplatin (or saline) administration, urine and blood samples were obtained and kidneys were removed.

\section{Preparation of DPPD-loaded Poly(lactide-co-glycolic acid) (PLGA) nanoparticles}

The DPPD-loaded PLGA nanoparticles was prepared using "single emulsion-solvent evaporation method" through a modified procedure. Briefly, $500 \mathrm{mg}$ of PLGA was dissolved in $10 \mathrm{ml}$ of suitable solvent. A solution of $100 \mathrm{mg}$ DPPD was added with stirring to the PLGA solution. Afterward, the PLGA/DPPD mixture was added dropwise with high vortexing to a surfactant solution. Once all of the PLGA/DPPD mixture was added, the contents was sonicated at $60 \%$ amplitude with a probe type sonicator (Misonix ultrasonic processor, S-4000, MisonixInc, CT, USA) to create an oil-in-water emulsion. Sonication process was carried out in an ice-water bath with using pulse function to prevent the heat built-up of the PLGA/DPPD solution during the sonication. After sonication, the emulsion was immediately poured into $100 \mathrm{ml}$ of diluted surfactant solution under rapid stirring. The resulting nanosized PLGA emulsion was then stirred to allow for solvents evaporation. The produced PLGA nanoparticles suspension was diluted to $150 \mathrm{ml}$ volume with de-ionized water and used for further investigations. The plain PLGA nanoparticles was synthesized using the same procedure but without the DPPD [11].

\section{Measurement of biochemical parameters}

- Blood samples were used for determination of serum creatinine, BUN, serum magnesium levels by standard laboratory methods and monocyte chemoattractant protein 1 (MCP-1) by ELISA according to the commericial kits Rat MCP-1 Quantikine, respectively (R\&D systems, Minneapolis,MN,USA).

- Urine samples were used for determination of urinary creatinine in order to calculate creatinine clearance by standard laboratory methods.

- Tissue samples were used for determination of total collagen [12].

- These parameters were measured using :

$>$ An automated spectrophotometer (Slim Plus, Italy).

$>$ Microplate Reader (Stat Fax 3200, Awareness).

\section{Histopathological examination}

Renal morphology: Kidneys were perfused in a retrograde fashion through the abdominal aorta using saline $0.9 \%$ till complete clearance of the perfusion fluid, and then $10 \%$ neutral buffered formalin for in situ fixation. Both kidneys in all groups were harvested, cut longitudinally, and send for pathological evaluation in $10 \%$ neutral buffered formalin. Samples were processed and embedded in paraffin wax and sections (4 $\mu \mathrm{m}$ thick) were evaluated for the following:

Tubular injury score: sections were stained with hematoxylin and eosin (H \& E) for light microscopic examination.

Total collagen and detection of interstitial fibrosis: renal tissue samples of the experiment were stained with Masson trichrome. This stain is used as routine connective tissue stain. It stains collagen blue while nuclei are stained red to blue

\section{Immunohistochemical staining for $\alpha$-SMA and Ki-67:}

- Detection of alpha smooth muscle actin ( $\alpha$-SMA): The degree of $\alpha$-SMA in all groups was assessed in renal tissue samples of the experiment using a specific kit that detect $\alpha$-SMA protein in rat cells (DakoCytomation, Glostrup, Denmark).

- Detection of proliferation marker Ki-67 antigen: The degree of cellular proliferation in all groups was assessed in renal tissue samples of the experiment using a specific kit that detect Ki-67 antigen in rat cells (DakoCytomation, Glostrup, Denmark).

\section{Statistical analysis}

Data were tabulated, coded then analyzed using the computer program SPSS (Statistical package for social science) version 17.0 to obtain descriptive data. Descriptive statistics were calculated in the form of mean \pm standard deviation (SD) and median, minimum and maximum. In the statistical comparison between the different groups, the significance of difference was tested using one of the following tests: ANOVA (analysis of variance): Used to compare between more than two groups of numerical (parametric) data followed by post-hoc tukey, Kruskal Wallis test: Used to compare between more than two groups of numerical (non-parametric) data followed by mann-whitney for pairwise comparisons or Repeated measures ANOVA (analysis of variance): Used to compare between more than two related groups of numerical (parametric) data followed by post-hoc LSD. A P value $<0.05$ was considered statistically significant.

\section{Results}

\section{At day 14}

Cisplatin group showed significant increase in serum creatinine, BUN and magnesium compared to control group $(\mathrm{p}<0.001)$ but Nano-DPPD group showed significant decrease in serum creatinine, BUN and magnesium compared to cisplatin group $(\mathrm{p}<0.05)$ as shown in Figures 1-3.

Also, cisplatin group showed significant decrease in creatinine clearance compared to control group $(p<0.001)$ but Nano-DPPD group showed significant increase in creatinine clearance compared to cisplatin group $(\mathrm{p}<0.001)$ as shown in Figure 4.

On the other hand, hydroxyproline content and MCP-1 were significantly increase in cisplatin group compared to control group ( $p$ $\leq 0.001$ ). But significantly decreased in Nano-DPPD group compared to cisplatin group $(\mathrm{p} \leq 0.001)$ as shown in Figures 5-6.

In case of Histopathological examination we found that at day 14 , tubular injury score and fibrosis score were significantly increase 


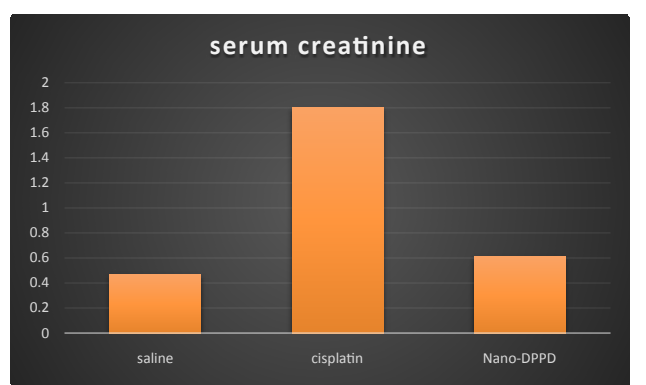

Figure 1. Effect of Nano-DPPD therapy on serum creatinine $(\mathrm{mg} / \mathrm{dl})$ in rat fibrosis model induced by cisplatin.

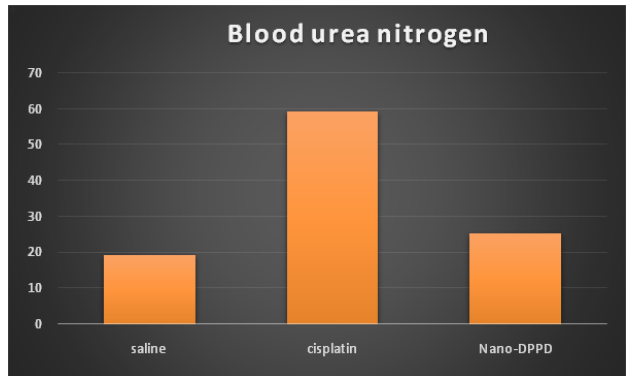

Figure 2. Effect of Nano-DPPD therapy on BUN (mg/dl) in rat fibrosis model induced by cisplatin.

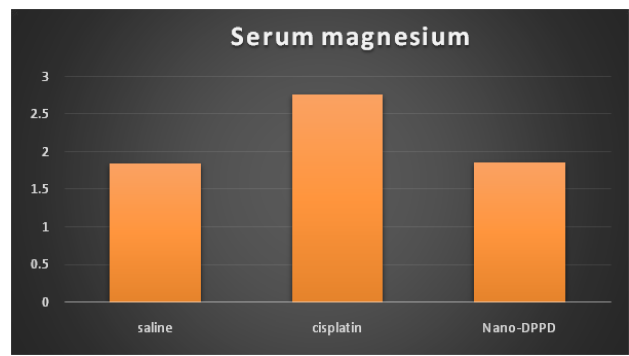

Figure 3. Effect of Nano-DPPD therapy on serum magnesium $(\mathrm{mg} / \mathrm{dl})$ in rat fibrosis model induced by cisplatin.



Figure 4. Effect of Nano-DPPD therapy on oncreatinine clearance (m1/min./100g body weight) in rat fibrosis model induced by cisplatin.

in cisplatin group compared to control group $(\mathrm{p} \leq 0.001)$. But NanoDPPD group showed significant decrease in tubular injury score and fibrosis score compared to positive group $(\mathrm{p}<0.05)$ as shown in Figures 7-9.

At day 14, ASMA-positive cells and Ki-67 proliferation marker were significantly increase in cisplatin group compared to control group ( $\mathrm{p} \leq 0.001)$. But significantly decreased in Nano-DPPD group compared to positive group $(\mathrm{p} \leq 0.001)$ as shown in Figures 10-12.

\section{Discussion}

The present study indicated that Nano-DPPD dramatically protected the cisplatin-induced in vivo nephrotoxicity in rat. However, the mechanisms underlying the cisplatin-induced acute renal failure have not been fully understood, several investigators have shown that the ROS or free radicals and collagen deposition are closely related to renal fibrosis induced by cisplatin [13].

Several studies have reported that the alterations induced by cisplatin in the kidney functions were characterized by signs of injury, such as increase in products of lipid peroxidation and changes in total collagen concentration in kidney tissue as well glucose, protein, creatinine and urea levels, in urine and plasma samples [14]. In the present study, it has been shown that administration of cisplatin to rat caused an elevation in total tissue collagen and serum chemoattractant protein $\mathrm{MCP}-1$, which correlated with increase in plasma creatinine, urea and magnesium levels. And also, correlated with decline in

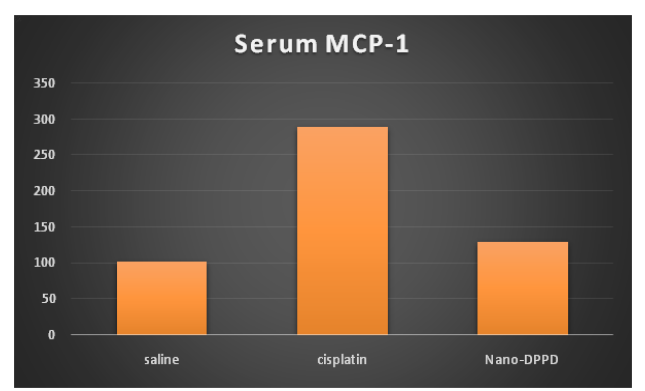

Figure 5. Effect of Nano-DPPD therapy on serum MCP-1 $(\mathrm{pg} / \mathrm{ml})$ in rat fibrosis model induced by cisplatin.



Figure 6. Effect of Nano-DPPD therapy on onhyroxyproline content (ug / mg kidney tissue) in rat fibrosis model induced by cisplatin.

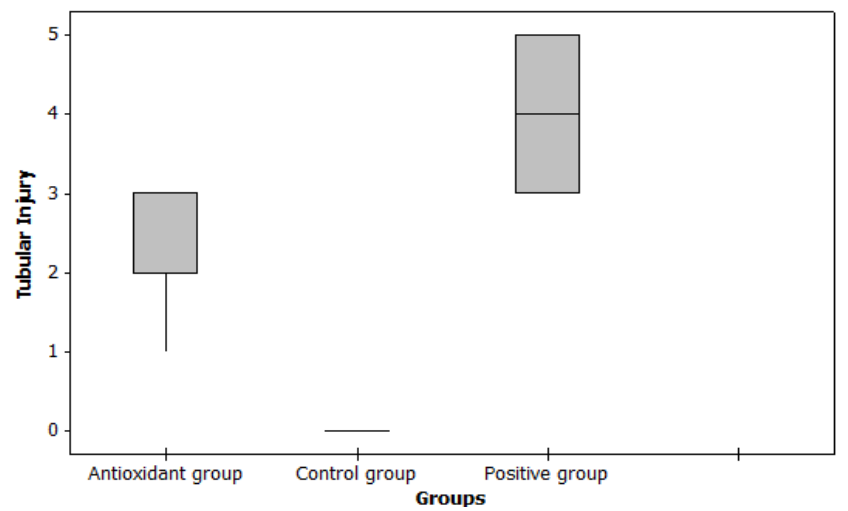

Figure 7. Effect of Nano-DPPD therapy on tubular injury in rat fibrosis model induced by cisplatin. 


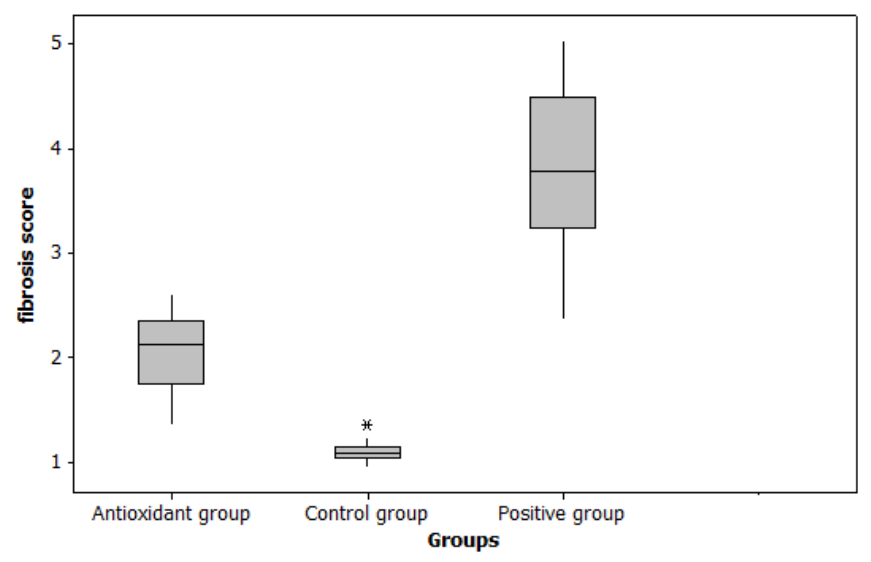

Figure 8. Effect of Nano-DPPD therapy on fibrosis score in rat fibrosis model induced by cisplatin.
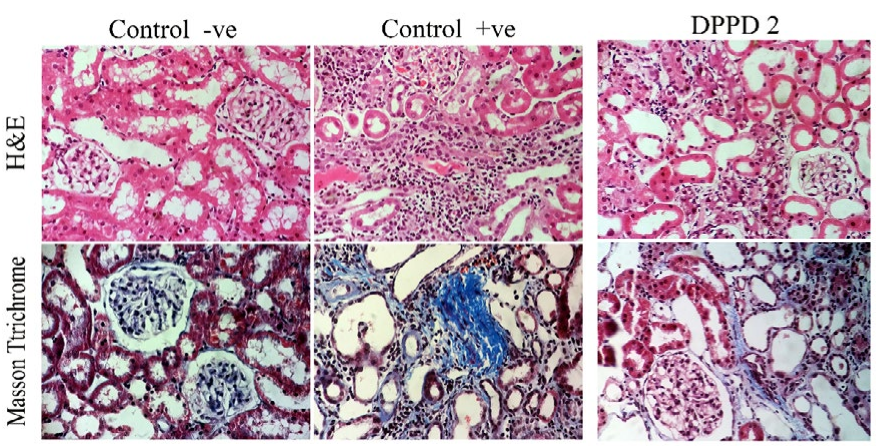

Figure 9. Histopathological examination (Tubular injury score - Total collagen and detection of interstitial fibrosis ) in all studied groups.

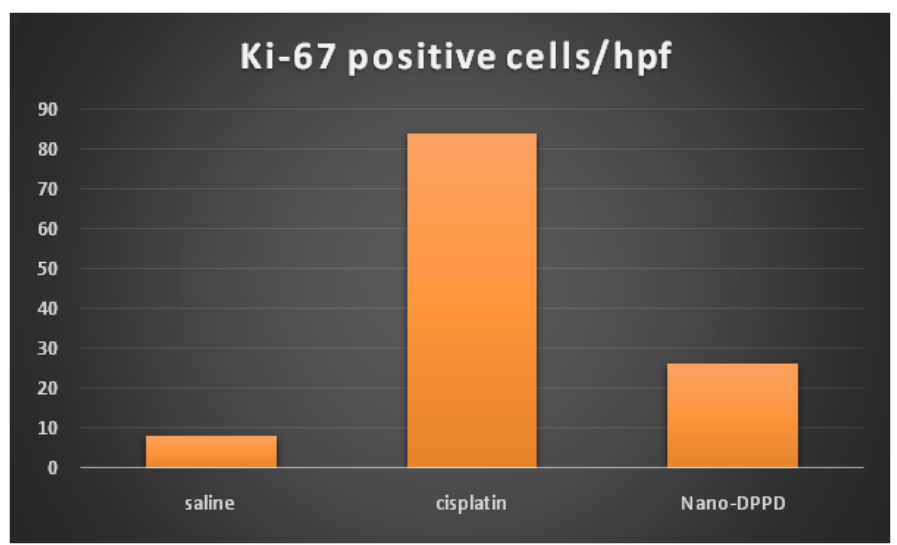

Figure 10. Effect of antioxidant and mesenchymal stem cell therapy on Ki-67 positive cells/HPF in rat fibrosis model induced by cisplatin.

creatinine clearance level Also, cisplatin-induced nephrotoxicity was accompanied by an increase in KI-67 proliferation marker, $\alpha$-smooth muscle actin in kidney tissue. These histopathological results were well correlated with the renal biochemical parameters. The current study demonstrates that Nano-DPPD provides protection against cisplatininduced acute renal failure in rat. Treatment with Nano-DPPD resulted in a lower level of creatinine, magnesium and urea in plasma and total tissue collagen and serum chemoattractant protein MCP-1 and increasing creatinine clearance level than cisplatin received group, indicating improvement in the renal function. The histopathological evaluation of the kidney preparations in Nano-DPPD - treated groups

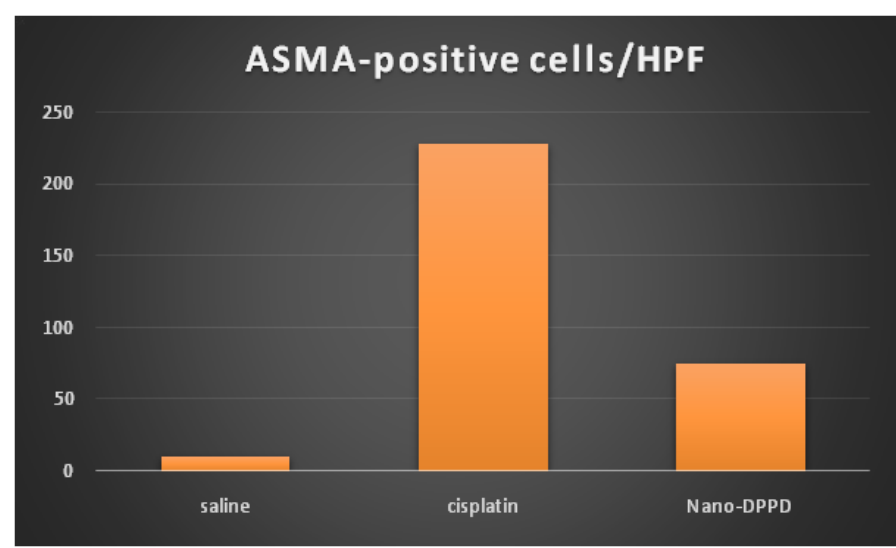

Figure 11. Effect of antioxidant and mesenchymal stem cell therapy on ASMA-positive cells/HPF in rat fibrosis model induced by cisplatin.
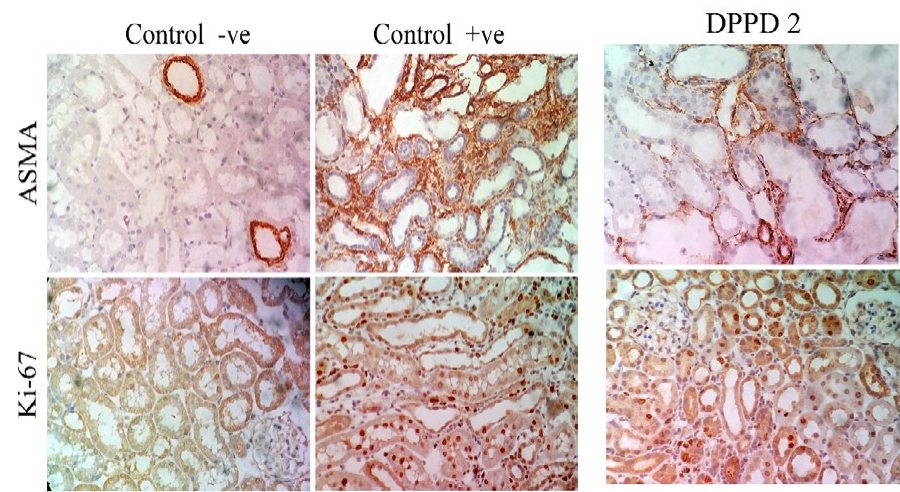

Figure 12. Immunohistochemical staining for $\alpha$-SMA and Ki-67in all studied groups

also revealed a decrease in cisplatin-induced renal injury and fibrosis score. Although the exact mechanism of cisplatininduced renal fibrosis is not well understood, several investigators have shown that cisplatin renal fibrosis is associated with increase the infilteration and proliferation of the myofibroblast, a specialized fibroblast characterized by cytoplasmic stress fibres with alpha smooth muscle actin (a-SMA) in renal tissue [14]. It has been suggested that binding of cisplatin to the renal base transport system and the following infiltration process is the main cause of renal fibrosis. There is evidence suggesting that cisplatin exerts its nephrotoxic effects by the generation of free radicals and increasing collagen deposition that results from infiltration of myofibroblast to interstitium [15]. In the present investigation, treatment with Nano-DPPD inhibited the increase alpha smooth muscle actin ( $\alpha$-SMA) induced by cisplatin in renal tissue and also, inhibited increasing in KI-67 proliferation marker. Nano-DPPD has reversed the enhancement of a SMA and KI-67 level to a considerable extent, thereby confirming its antioxidant role in cisplatin acute renal failure [16].

In conclusion, Nano-DPPD is able to protect the kidneys against cisplatin-induced fibrosis. But, before a conclusive statement on potential usefulness of Nano-DPPD as adjunct to the cisplatin therapy, there is a need for further studies including human trials.

\section{Acknowledgements}

The authors would extend their thanks to the whole technicians in the Medical Experimental Research Centre, Faculty of Medicine, 
Mansoura University and in Zewail City for Science and Technology for their great dedicated work.

\section{Disclosures}

No conflicts of interest, financial or otherwise, are declared by the authors.

\section{References}

1. Dobyan DC, Levi J, Jacobs C, Kosek J, Weiner MW (1980) Mechanism of cis-platinum nephrotoxicity: II. Morphologic observations.J Pharmacol Exp Ther 213: 551-556. [Crossref]

2. Chopra S, Kaufman JS, Jones TW, Hong WK, Gehr MK, Hamburger RJ, et al. (1982) Cis-diamminedichloroplatinum-induced acute renal failure in the rat. Kidney Int 21: 54-64.

3. Gemba M, Fukuishi N, Nakano S (1988) Effect of N-N'-diphenyl-pphenylenediamine pretreatment on urinary enzyme excretion in cisplatin nephrotoxicity in rats. Jpn $J$ Pharmacol 46: 90-92.[Crossref]

4. Antunes LM, Darin JD, Bianchi MD (2000) Protective effects of vitamin C against cisplatin-induced nephrotoxicity and lipid peroxidation in adult rats: a dose-dependent study. Pharmacol Res 41: 405-411.[Crossref]

5. Nakano S, Gemba M (1989) Potentiation of cisplatin-induced lipid peroxidation in kidney cortical slices by glutathione depletion. Jpn J Pharmacol 50: 87-92.[Crossref]

6. Tsutsumishita Y, Onda T, Okada K, Takeda M, Endou H, Futaki S, et al. (1998) Involvement of $\mathrm{H} 2 \mathrm{O} 2$ production in cisplatin-induced nephrotoxicity. BiochemBiophys Res Commun 242: 310-312. [Crossref]

7. Guinee DG Jr, van Zee B, Houghton DC (1993)Clinically silent progressive renal tubulointerstitial disease during cisplatin chemotherapy. Cancer 71: 4050-4054. [Crossref]

8. Dobyan DC (1985) Long-term consequences of cis-platinum-induced renal injury: a structural and functional study. AnatRec 212: 239-245.[Crossref]

9. Nangaku M (2004) Mechanisms of tubulointerstitial injury in the kidney final common pathways to end-stage renal failure. Intern Med 43: 9-17.[Crossref]

10. Sugihara K, Nakano S, Koda M, Tanaka K, Fukuishi N, et al. (1987) Stimulatory effect of cisplatin on production of lipid peroxidationin renal tissues. Jpn J Pharmacol 43: 247-252.[Crossref]

11. Hirata IY, Cezari MHS, Nakaie CR, Boschcov P, Ito AS, et al. (1999) Internally quenchedfluorogenic protease substrates: solid phase synthesis and fluorescence spectroscopy of peptides containing ortho-amino benzoyl/dinitrophenyl groups as donor-acceptor pairs. Letters in Peptide Science 1: 299-308.

12. Prockop DJ, Udenfriend S (1960) A specific method for the analysis of hydroxyproline in tissues and urine.Anal Biochem 1: 228-239.[Crossref]

13. Sueishi K, Mishima K, Makino K, Itoh Y, Tsuruya K, et al. (2002) Protection by a radical scavenger edaravone against cisplatin-induced nephrotoxicity in rats. Eur. $J$ Pharmacol 451: 203-208. [Crossref]

14. Atessahin A,Yilmaz S., Karahan I, Ceribasi AO,Karaoglu A (2005) Effects of lycopene against cisplatin-induced nephrotoxicity and oxidative stress in rats. Toxicology 212: 116-123.[Crossref]

15. Baek SM, Kwon CH, Kim JH, Woo JS, Jung JS, et al. (2003) Differential roles of hydrogen peroxide and hydroxyl radical in cisplatininduced cell death in renal proximal tubular epithelial cells. J Lab Clin Med 142: 178-186.[Crossref]

16. Ochiai T, Soeda S, Ohno S, Tanaka H, Shoyama Y, et al. (2004) Crocin prevents the death of PC-12 cells through sphingomyelinase-ceramide signaling by increasing glutathione synthesis. Neurochem Int 44: 321-330.[Crossref]

Copyright: (C2016 Zahran F. This is an open-access article distributed under the terms of the Creative Commons Attribution License, which permits unrestricted use, distribution, and reproduction in any medium, provided the original author and source are credited. 